\title{
Diffusion in a quasi-one-dimensional system on a periodic substrate
}

\author{
J. C. N. Carvalho, ${ }^{1,2, *}$ K. Nelissen,,${ }^{2,1, \dagger}$ W. P. Ferreira, ${ }^{1, \ddagger}$ G. A. Farias, ${ }^{1, \S}$ and F. M. Peeters ${ }^{2,1, \|}$ \\ ${ }^{1}$ Departamento de Física, Universidade Federal do Ceará, Caixa Postal 6030, Campus do Pici, 60455-760 Fortaleza, Ceará, Brazil \\ ${ }^{2}$ Department of Physics, University of Antwerp, Groenenborgerlaan 171, BE-2020 Antwerpen, Belgium \\ (Received 9 June 2011; revised manuscript received 21 December 2011; published 23 February 2012)
}

\begin{abstract}
The diffusion of charged particles interacting through a repulsive Yukawa potential, $\exp (-r / \lambda) / r$, confined by a parabolic potential in the $y$ direction and subjected to a periodic substrate potential in the $x$ direction is investigated. Langevin dynamic simulations are used to investigate the effect of the particle density, the amplitude of the periodic substrate, and the range of the interparticle interaction potential on the diffusive behavior of the particles. We found that in general the diffusion is suppressed with increasing the amplitude of the periodic potential, but for specific values of the strength of the substrate potential a remarkable increase of the diffusion is found with increasing the periodic potential amplitude. In addition, we found a strong dependence of the diffusion on the specific arrangement of the particles, e.g., single-chain versus multichain configuration. For certain particle configurations, a reentrant behavior of the diffusion is found as a function of the substrate strength due to structural transitions in the ordering of the particles.
\end{abstract}

DOI: 10.1103/PhysRevE.85.021136

PACS number(s): 05.40.Jc, 66.10.C-, 82.70.Dd

\section{INTRODUCTION}

Diffusion can be defined as the movement of particles between regions of different concentration; however, it can also occur even when there is no concentration gradient applied. As examples of diffusion in terms of many particles we can cite heat diffusion and molecular diffusion, that is, a net transport of molecules from a region of higher concentration to one of lower concentration by random molecular motion. Diffusion can also be discussed in terms of the movement of one particle, for example, we have the Brownian motion [1], which is the random movement of microscopic particles suspended in a liquid or gas, caused by collisions with the surrounding medium.

Particle transport in very restricted geometries was first studied by Harris in 1965 [2]. He considered one-dimensional (1D) hardcore particles diffusing along a line where mutual passage is prohibited. Consequently, the sequence of particles remains unaffected during time (single-filing condition). In this system particles are strong correlated in order that the displacement of individual particles drives the displacement of the other particles along the same direction. This phenomenon became known as single-file diffusion (SFD). The no passage restriction causes fundamental changes in the diffusive behavior.

In the last decades, SFD has been extensively studied due to its numerous applications in biology, chemistry, and physics. Some examples are the flow of ions, colloids, and water through molecular-sized channels in membranes [3-5], molecular sieving effects due to transport of adsorbate molecules through micropores [6], colloidal transport in quasi-onedimensional (Q1D) channels [7-9], and nanofluidic separation of rigid macromolecules (DNA) through a nanofilter [10]. One important property of SFD is that the mean-square displacement (MSD) $W(t)$ of a particle, for a time $t \gg \tau$ (i.e.,

\footnotetext{
*joaoclaudio@fisica.ufc.br

${ }^{\dagger}$ kwinten.nelissen@ua.ac.be

${ }_{\ddagger}$ wandemberg@ fisica.ufc.br

§gil@fisica.ufc.br

"francois.peeters@ua.ac.be
}

the time that one particle needs to move a significant fraction of the mean particle distance), is given by

$$
\lim _{t \gg \tau} W(t)=2 F \sqrt{t},
$$

where $F$ is the SFD mobility, $t$ is the time, and $W(t)=\left\langle\Delta x^{2}\right\rangle$. SFD processes, in contrast to two-dimensional (2D) and three-dimensional (3D) self-diffusion, cannot be described by a diffusion coefficient and therefore does not obey Fick's law. This non-Fickian behavior is a fundamental property of SFD, which does not lead to a linear increase of $W(t)$ with time, and is therefore called anomalous diffusion. For normal diffusion, the diffusion coefficient $D$ is defined by the relation $\left\langle\Delta x^{2}\right\rangle=2 D t$. This relation is valid up to a characteristic time, after which particle collisions become important and subdiffusive behavior in the form of Eq. (1) appears.

In a recent work of Kollmann [11] it was shown that $W(t)$ is determined by the short-time and large-scale collective behavior of the particles in the system. Equation (1) holds for any kind of interaction potential as long as the correlation length between the particles is finite and it is valid for any physical system in the fluid state. Furthermore, this relation is unique in the case of classical systems and overdamped systems.

The first experimental attempts to observe Eq. (1) were realized in zeolitic materials, which consist of long quasicylindrical pores with diameters of several angstroms [12]. Zeolite molecular sieves (see, e.g., Ref. [13]) are characterized by selective adsorption due to the uniform pore size of the zeolite structure and high adsorption capacity for polar substances at low concentrations. Molecular diffusion of tetrafluoromethane in zeolite $\mathrm{AlPO}_{4}-5$ was studied by pulsed-field gradient NMR spectroscopy. The channel diameter, obtained from a structural analysis by $\mathrm{x}$-ray diffraction, is of the order of $0.73 \mathrm{~nm}$, whereas the diameter of the $\mathrm{CF}_{4}$ molecules is $0.47 \mathrm{~nm}$. Thus the mutual passage of molecules is excluded. In a zeolite channel the movement of an isolated particle, after a short ballistic period, is determined by the stochastic interaction with the channel walls producing diffusional anomalous behavior [14]. The experimental evidence for the 
occurrence of SFD as provided by different authors remained contradictory for some time [15]. The anomalous diffusive behavior was found by Wei et al [8], who investigated the dynamics of paramagnetic colloids in a Q1D channel. Several analytical models have been proposed for the study of SFD [16,17]. SFD of interacting particles is numerically investigated in Ref. [9]. The authors show that SFD depends on the interparticle interaction and could be suppressed if the interaction is strong enough, displaying even a subdiffusive behavior slower than $t^{1 / 2}$. A Langevin formulation for SFD [18] is presented in Ref. [19].

A 2D system is often created in the presence of a substrate, which may induce a periodic potential on the particles. The present model system of Yukawa particles can be realized experimentally using, for example, dusty plasma. A dusty plasma consists of interacting microscopic dust particles immersed in an electron-ion plasma. Dust particles can be confined to a 2D layer through a combination of gravitational and electrical forces. By microstructuring a channel in the bottom electrode of the discharge, it is possible to laterally confine the dust particles, as was realized experimentally in Ref. [20,21]. The strength of the $1 \mathrm{D}$ confinement potential can be varied by the width of the channel or the potential on the bottom electrode. When the width of the channel is microstructured into an oscillating function along the channel, it will result in a periodic potential along the channel.

In this paper we study the diffusion of charged particles in a Q1D system subjected to a periodic substrate. By increasing the amplitude of the substrate modulation, we observe a transition from normal diffusion, $W(t) \propto t$, at short times to subdiffusion at intermediate times, $W(t) \propto t^{\alpha}$, with $\alpha<1$, which depends on the amplitude of the periodic potential. For certain values of the particle density a nonmonotonic dependence of $\alpha$ on the amplitude of the periodic potential is found. For long times normal diffusion is recovered, i.e., $W(t) \propto t$. A typical example of the time dependence of $W(t)$ is shown in Fig. 1 together with the three different diffusion regimes.

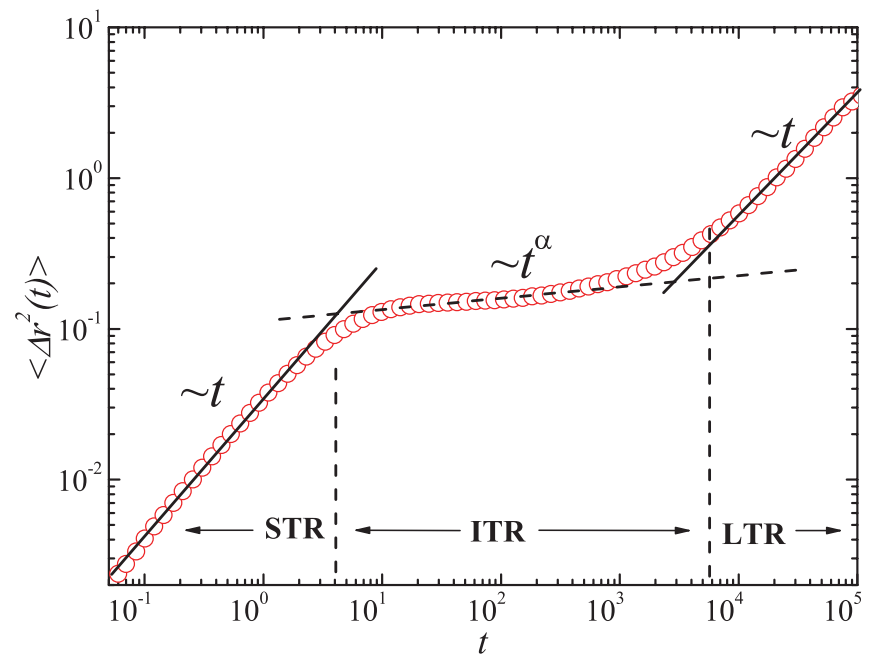

FIG. 1. (Color online) Example of the time dependence of the mean-square displacement (red circles curve) and indication of the three different regimes whose characteristic time dependence is shown by the dashed lines.
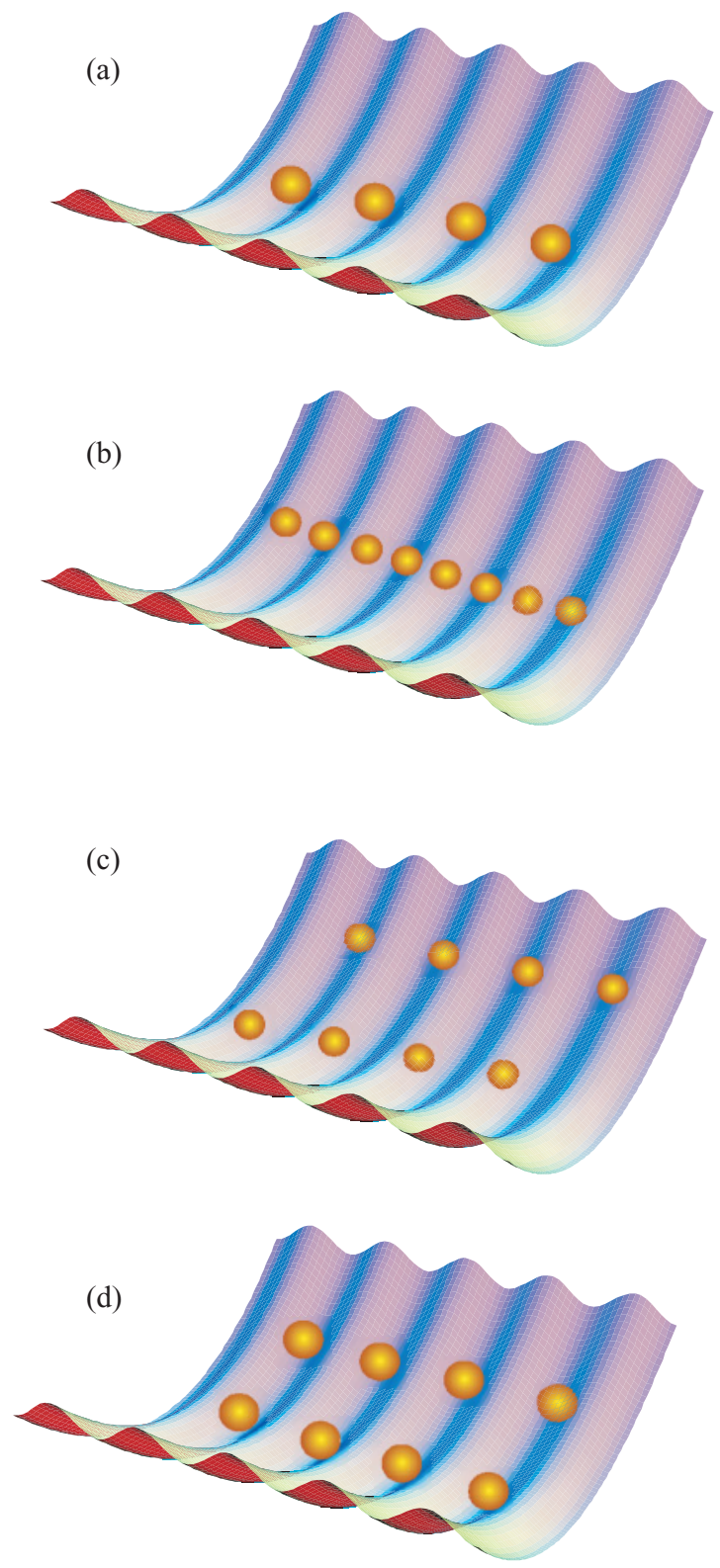

FIG. 2. (Color online) Sketches of the model system for the following configurations: (a) one chain, with one particle positioned at each minimum of the periodic potential; (b) one chain, with two particles positioned at each minimum; (c) two chains, with particles in a staggered configuration; and (d) two chains with particles in an aligned configuration.

In a recent work [22] we studied the zero temperature ground state structures and normal mode spectra of the model system proposed in this paper. That is, we investigated a classical 2D system of charged particles interacting through a repulsive Yukawa potential, $e^{-r / \lambda} / r$, and confined in a parabolic channel which limits the motion of the particles in the $y$ direction. Along the $x$ direction, the particles are subjected to a periodic potential. Due to the parabolic confinement the system self-organizes in chains (rows) along the $x$ direction. We found that the number of chains is strongly dependent on the density and the intensity of the periodic potential $V_{0}$.

The structures are mainly ruled by the fact that particles tend to move to the minima of the periodic substrate potential, 
modifying the symmetry of the ordered structures. However, for small $V_{0}$, the interparticle repulsive interaction dominates and we found that it is possible to find particles at arbitrary positions of the periodic potential, including regions near to the maxima. For small densities the system can be found in a configuration with one or two chains (depending on $V_{0}$ ), as shown schematically in Fig. 2.

In the present work, we will study diffusion of the system as function of the strength of the substrate for three particular configurations: (i) the single-chain configuration, where the particles are aligned on a single line in the minimum of the confinement potential; (ii) the two-chain configuration, where the particles are organized in two distinguishable rows; and (iii) a substrate-dependent configuration, where structural transitions from the single-chain to the two-chain configuration can occur by changing the strength of the substrate [Figs. 2(c) and $2(\mathrm{~d})]$.

The paper is organized as follows. In Sec. II we present the model system and our numerical approach. In Sec. III we give our numerical results and investigate in more detail the intermediate time regime and its dependence on the particle density and the amplitude of the periodic potential. Finally in Sec. IV our conclusions are formulated.

\section{MODEL SYSTEM AND NUMERICAL APPROACH}

Our system consists of identical pointlike charged particles interacting through a screened Coulomb potential (Yukawa potential). The particles are restricted to move in a $2 \mathrm{D}$ plane and are subjected to an external parabolic confinement in the $y$ direction and a periodic substrate potential along the $x$ direction. The diffusion of the colloidal particles moving in a nonmagnetic liquid is overdamped and the stochastic Langevin equations of motion can be reduced to those for Brownian particles [23]:

$$
\frac{d \vec{r}_{i}}{d t}=-\frac{D}{k_{B} T}\left(\sum_{j=1}^{N} \frac{d V_{\mathrm{int}}\left(\vec{r}_{i j}\right)}{d \vec{r}_{i}}+\frac{d V_{c}\left(\vec{r}_{i}\right)}{d \vec{r}_{i}}+\frac{d V_{\mathrm{sub}}\left(\vec{r}_{i}\right)}{d \vec{r}_{i}}-\vec{F}_{T}^{i}\right),
$$

where $\vec{r}_{i}$ and $D$ are the position and the self-diffusion coefficient, respectively, $t$ is the time, $k_{B}$ is the Boltzmann constant, and $T$ is the absolute temperature of the system.

Further $V_{c}\left(\vec{r}_{i}\right)$ stands for the confining potential with $\vec{r}_{i}=\left(x_{i}, y_{i}\right) ; V_{\text {int }}\left(r_{i j}\right)$ for the interaction energy between the particles, where $r_{i j}=\left|\vec{r}_{i}-\vec{r}_{j}\right|$ is the distance between the $i$ th and $j$ th particle; and $V_{\text {sub }}\left(\vec{r}_{i}\right)$ for the substrate potential. The confinement potential is taken to be

$$
V_{c}\left(\vec{r}_{i}\right)=\frac{1}{2} m \omega_{0}^{2} y_{i}^{2},
$$

where $m$ is the mass of each particle, $\omega_{0}$ is the strength of the parabolic confining potential, and $y_{i}$ is the distance of the $i$ th particle from the central axis of the confinement along the $x$ direction. The particles interact through a screened Coulomb potential given by

$$
V_{\text {int }}\left(r_{i j}\right)=\frac{q^{2}}{\epsilon} \frac{e^{-\left|\vec{r}_{i}-\vec{r}_{j}\right| / \lambda}}{\left|\vec{r}_{i}-\vec{r}_{j}\right|},
$$

where $q$ is the charge of each particle, $\epsilon$ is the dielectric constant of the medium, and $\lambda$ is the Debye screening length. The periodic substrate potential acting on each particle, $V_{\text {sub }}\left(\vec{r}_{i}\right)$, is given by

$$
V_{\mathrm{sub}}\left(\vec{r}_{i}\right)=V_{0} \sum_{i} \cos \left(\frac{2 \pi x_{i}}{L}\right),
$$

where $V_{0}$ is the strength of the periodic substrate potential and $L$ its periodicity.

$\vec{F}_{T}^{i}$ is a randomly fluctuating force, with the following properties: $\left\langle\vec{F}_{T}\right\rangle=0$ and $\left\langle F_{T}^{i}(t) F_{T}^{i^{\prime}}\left(t^{\prime}\right)\right\rangle=2 \eta k_{B} T \delta_{i i^{\prime}} \delta\left(t-t^{\prime}\right)$, which means that it is normally distributed (i.e., Gaussian distribution), where $\eta$ is the friction coefficient. Taking the same units of energy and distance from Ref. [22], i.e., $E_{0}=\left(m \omega_{0}^{2} q^{4} / 2 \epsilon^{2}\right)^{1 / 3}$ and $r_{0}=\left(2 q^{2} / \epsilon m \omega_{0}^{2}\right)^{1 / 3}$, Eq. (2) can be rewritten in a dimensionless form as

$\frac{d{\overrightarrow{r^{\prime}}}_{i}}{d t^{\prime}}=-\left(\Omega \sum_{j=1}^{N} \frac{d V_{\mathrm{int}}^{\prime}\left({\overrightarrow{r^{\prime}}}_{i j}\right)}{d \vec{r}_{i}^{\prime}}+\frac{d V_{c}^{\prime}\left(\vec{r}_{i}^{\prime}\right)}{d{\overrightarrow{r^{\prime}}}_{i}}+\frac{d V_{\mathrm{sub}}^{\prime}\left(\vec{r}_{i}^{\prime}\right)}{d{\overrightarrow{r^{\prime}}}_{i}}-\vec{F}^{\prime}{ }_{T}^{i}\right)$,

where the screening parameter is defined as $\kappa=r_{0} / \lambda$ and $\Omega=q^{2} / k_{B} T \epsilon r_{0}$. The unit of length is defined as the distance at which the confinement energy equals the Coulomb energy. The time $t^{\prime}$ is given in units of $\omega_{0}^{-1}$. The self-diffusion coefficient is in units of $r_{0}^{2} \omega_{0}$ and the temperature is measured in units of $T_{0}=E_{0} / k_{B}$.

For the numerical integration of the equations of motion we use a first-order finite difference method with periodic boundary conditions along the $x$ direction and a time step $\Delta t=0.0005$, and for the screening parameter we take $\kappa=1$, which is a typical value for dusty plasma and colloidal systems. In the numerical simulations, we typically consider $300-400$ particles in our computational unit cell. The simulations were performed for $\Omega=10$ and $\Omega=100$, which correspond to a weakly correlated situation and a strongly correlated situation, respectively. Further, the system is defined by $V_{0}, L$, and the particle density $n$, which is the ratio between the number of chains $N_{\text {ch }}$ and $a_{0}$, i.e., $n=N_{\text {ch }} / a_{0}$, where $a_{0}$ is the average separation between particles along the chain, calculated when the substrate is not present [22], i.e., when $V_{0}=0$. Examples of particle positions taken over a large time interval are shown in Fig. 3 for different values of $V_{0}, n$, and $L / a_{0}$. The singleand two-chain configurations are shown. A melted situation [Fig. 3(a)] is also shown, where the particles move randomly between two chains.

\section{RESULTS AND DISCUSSIONS}

In general, particle transport or diffusion on a periodic substrate has been reported to be slower than the diffusion in the absence of it and its magnitude depends on the energetic balance between the particle-particle and particle-substrate interactions [24,25]. In this paper we show that such a behavior 

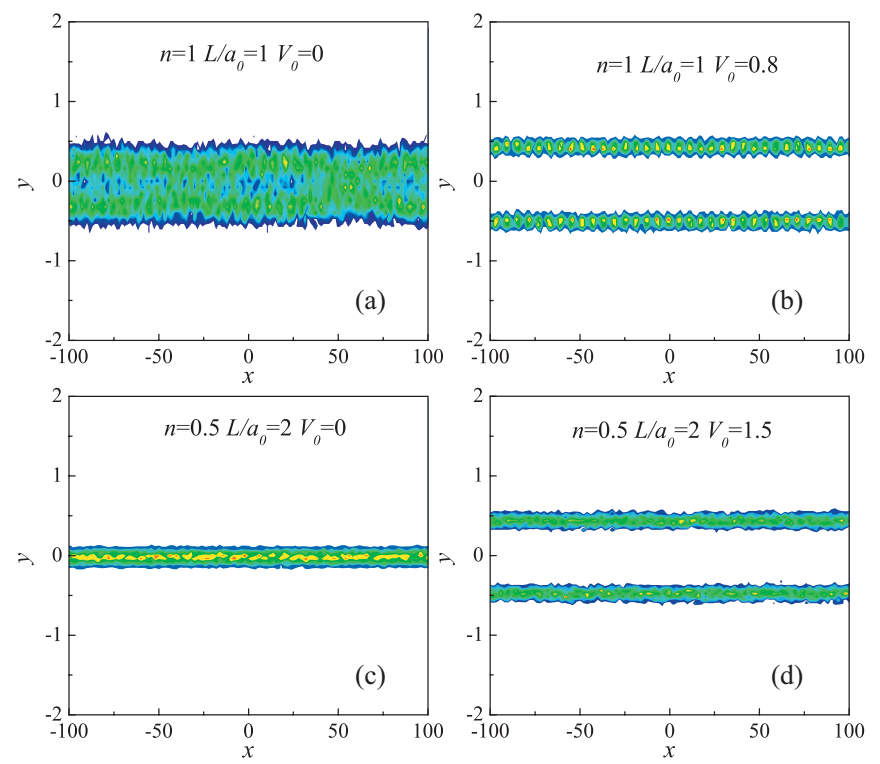

FIG. 3. (Color online) Two-dimensional plots of the particle positions during $5 \times 10^{8}$ time steps for $\Omega=100$ considering (a) $n=1.0, L / a_{0}=1.0$, and $V_{0}=0.0$; (b) $n=1.0, L / a_{0}=1.0$, and $V_{0}=0.8$; (c) $n=0.5, L / a_{0}=2.0$, and $V_{0}=0.0$; and (d) $n=0.5$, $L / a_{0}=2.0$, and $V_{0}=1.5$.

is not always found in a Q1D system. Further, structural transitions can occur depending on the periodicity of the substrate and the particle density, which will have an impact on the diffusive behavior of the particles.

In order to characterize the diffusion of the system, we calculate the MSD as follows:

$$
\left\langle\Delta r^{2}(t)\right\rangle=\frac{1}{N}\left\langle\sum_{i=1}^{N}\left[r_{i}\left(t_{0}+t\right)-r_{i}\left(t_{0}\right)\right]^{2}\right\rangle_{\Delta t^{*}},
$$

where $r$ is the position of the particles, $N$ is the total number of particles, $t_{0}$ is the initial time, and $\langle\cdots\rangle_{\Delta t^{*}}$ represents a time average over a long time interval $\Delta t^{*}$ for different $t_{0}$ so that $0<t_{0}<\left(\Delta t^{*}-t\right)$ and $\Delta t^{*}$ is chosen to be equal to the total simulation time. Typically we consider $\Delta t^{*} \approx 2 \times 10^{9}$. Further $t_{0}$ was chosen to be equal to a multiple of $t$.

In order to illustrate our results we present the diffusion of the system as a function of the strength of the substrate for three particular configurations, namely: (i) the single-chain configuration, where the particles are aligned along a single row [Fig. 2(a)]; (ii) the two-chain configuration, where the particles are organized in two distinguishable rows [Figs. 2(c) and 2(d)]; and (iii) a substrate-dependent configuration, where structural transition from the single- chain configuration [Fig. 2(b)] to the two-chain configuration [Fig. 2(c)] can occur by changing the strength of the substrate. Further we analyze the above systems in the liquidlike state and for different values of $V_{0}$ (strength of the periodic potential). Please note that the coupling parameter $\Gamma=\langle V\rangle /\langle K\rangle$ :

$$
\Gamma=\frac{\langle V\rangle}{\langle K\rangle}=\Omega \frac{e^{-\kappa a_{0}}}{a_{0}},
$$

where $a_{0}$ is the average interparticle distance. For our numerical calculations we took $\kappa=1$ and we have typically $a_{0} \sim 1$ which results in $\Gamma \sim \Omega / e \sim 0.4 \Omega$. Further we consider two situations $\Omega=10$ and $\Omega=100$ which result in a $\Gamma$ value that is clearly in the liquidlike regime.

Three different time regimes can be distinguished. A typical example is provided in Fig. 1: (i) the short time regime (STR), which is governed by the overdamped motion of the particles resulting in $\left\langle\Delta r^{2}(t)\right\rangle \sim t$, where the $\mathrm{MSD}$ is $\left\langle\Delta r^{2}(t)\right\rangle<$ $\left(b . a_{0}\right)^{2}$ with $b$ being a parameter of order $0.1-1$; (ii) the intermediate time diffusion regime (ITR), where $\left\langle\Delta r^{2}(t)\right\rangle \sim$ $t^{\alpha}$, with $\alpha \leqslant 1$, and typically $\left(b \cdot a_{0}\right)^{2}<\left\langle\Delta r^{2}(t)\right\rangle<L^{2}$; and (iii) the long time regime (LTR), $\left\langle\Delta r^{2}(t)\right\rangle>L^{2}$, where the motion of the particles becomes fully correlated [9]. The translational invariance of the system results in a $\left\langle\Delta r^{2}(t)\right\rangle \sim$ $t$ behavior of overdamped motion. Since we are studying an infinite system (periodic boundary conditions in the $x$ direction) with a finite number of particles, we do not consider the very long time behavior, but instead we focus our research on the more interesting ITR. The MSD in the LTR is indicative of whether the particles are localized or not in the minima of the substrate, but are not representative for the diffusion in the long time limit since it depends on the total number of particles considered (i.e., the size of our unit cell) [17]. In the STR, the particles do not feel the presence of each other and perform a random walk, which causes a normal diffusion behavior. This regime has been intensively studied before and therefore is not discussed here. For the three regimes we fitted a $t^{\alpha}$ dependence, where $\alpha$ was determined such that the simulation results coincide with the $t^{\alpha}$ curve in the middle of the ITR.

\section{A. Single-chain configuration}

Inspired by our previous work on the ground state configuration of charged colloids subjected to a periodic substrate [22], we start with the simplest case where $n=0.5$ and $L / a_{0}=1.0$ for which a single-chain configuration was found as the ordered phase. For this parameter set, exactly one particle is positioned at each minimum of the periodic potential. Therefore, the configuration is not affected by the substrate strength.

In Figs. 4(a) and 4(b), the MSD is plotted as a function of time for $n=0.5$ and $L / a_{0}=1.0$ for $\Omega=10$ and $\Omega=100$. The slope $\alpha$ of the curves in the ITR is plotted as a function of $V_{0}$ (see insets in Figs. 4(a) and 4(b), respectively).

In the ITR the dynamical process becomes subdiffusive. When the strength of the substrate is zero $\left(V_{0}=0\right)$, the MSD curves [Figs. 4(a) and 4(b)] are characterized by a subdiffusive behavior, $\left\langle\Delta r^{2}(t)\right\rangle \propto t^{1 / 2}$, which is identified as SFD. If one increases the strength of the substrate $\left(V_{0}>0\right)$, the diffusion of particles is severely affected. The diffusion process in this regime becomes now slower than $\left\langle\Delta r^{2}(t)\right\rangle \propto t^{1 / 2}$. The observed reduction in the particle diffusion is caused by the energetic barrier imposed by the substrate. The particles oscillate around the substrate minima for a long time before reaching diffusive motion. Next, after some time, the MSD curves recover their linear increasing behavior. If one increases the substrate strength further, the particles become pinned at the minima of the substrate potential. That is the reason why the slope of the MSD curves in the ITR becomes zero for both $\Omega=10$ and $\Omega=100$, resulting in $\alpha=0$ (see insets of Fig. 4). The pinning of particles happens at much lower $V_{0}$ values for 

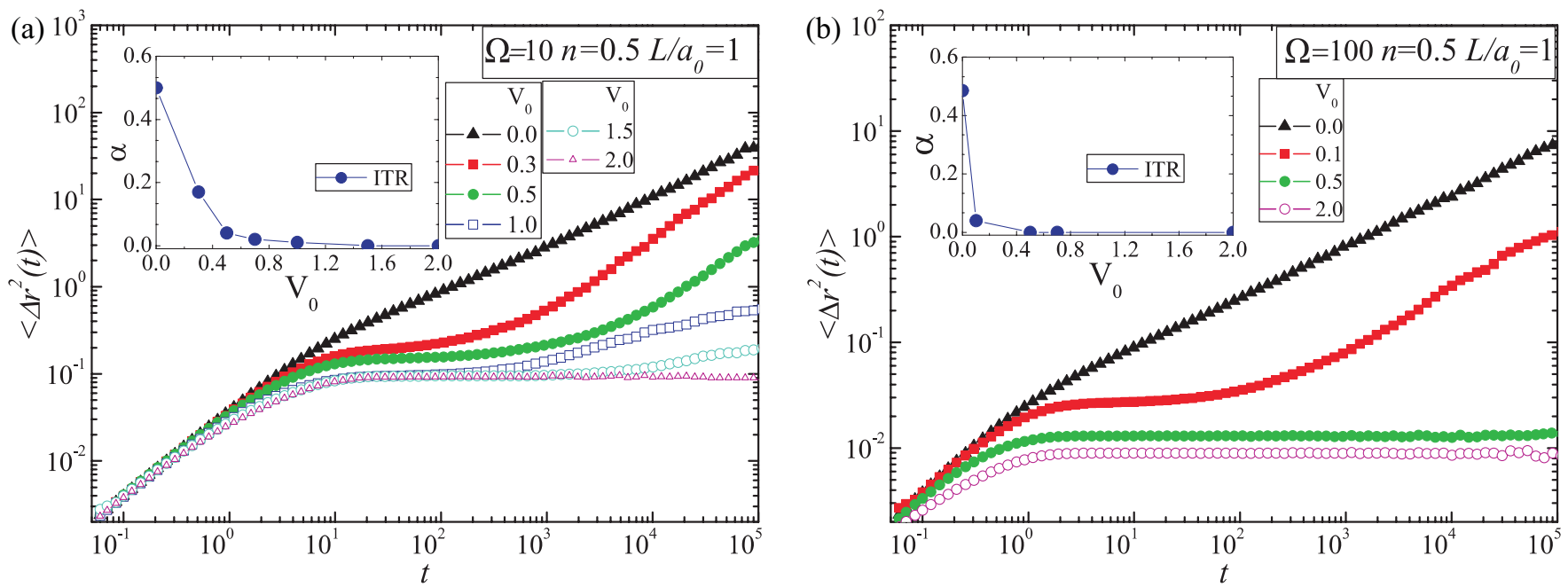

FIG. 4. (Color online) Log-log plot of the MSD curves taking into account both $x$ and $y$ directions, plotted as a function of time for $n=0.5$ and $L / a_{0}=1.0$ considering (a) $\Omega=10$ and (b) $\Omega=100$. The power-law coefficient $\alpha$ for the ITR is plotted as a function of $V_{0}$ in the insets.

$\Omega=100$ than for $\Omega=10\left(V_{0} \approx 0.5\right.$ versus $\left.V_{0} \approx 1.0\right)$ because of the weaker $\Omega$ of the system (see Fig. 4) in the latter case.

From this subsection we can conclude that increasing the amplitude of the substrate potential will reduce the mobility of the particles, which at a critical value of the substrate strength becomes pinned at the minima of the substrate potential. The critical $V_{0}$ value depends strongly on the parameter $\Omega$. Larger $\Omega$ values result in smaller critical $V_{0}$ values. A power-law dependence of $\Omega$ on $V_{c}$ was found and is presented in Fig. 5.

\section{B. Two-chain configuration}

Previously we found that, at zero temperature with $n=1.0$ and $L / a_{0}=1$, the particle configuration consists of two chains where its internal structure strongly depends on the strength of the periodic substrate $V_{0}$ [22]. In Fig. 4 of Ref. [22] the relevant internal distances for the ground state configuration are presented as a function of $V_{0}$. There it was found that,

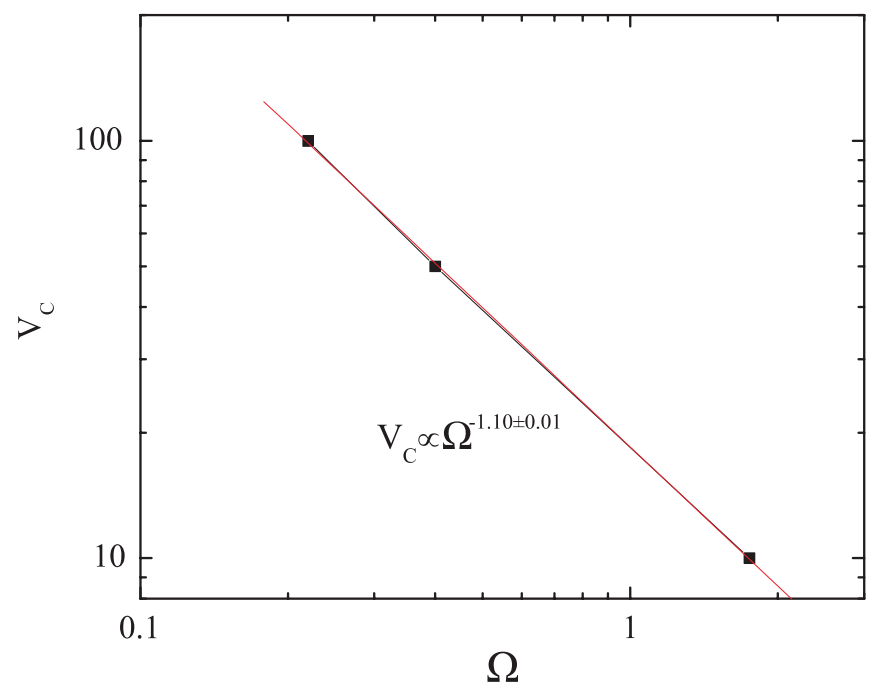

FIG. 5. (Color online) Critical value of $V_{0}$ for different values of $\Omega$. The curve is a power-law fit to our results. for $V_{0}=0.16$, the system undergoes a structural transition from a staggered [inset (I) Fig. 6(a)] to an aligned [inset (II) Fig. 6(a)] two-chain configuration, through a second-order (or continuous) structural transition.

For temperatures different from zero, the parameter $\Omega$ increases with increasing $V_{0}$ since the particles become closer to each other in each minimum of the periodic potential. Consequently the mean interaction energy between the particles is larger. In this case, $V_{0}$ can be seen as the inverse of an effective temperature, in order that an increase of $V_{0}$ will bring the system to a more ordered state. To illustrate that, we show in Figs. 3(a) and 3(b) the probability distributions of finding a particle at a certain position in the channel for $n=1.0$ and $L / a_{0}=1.0$. Warmer colors correspond with a higher probability. Notice that when the substrate strength is sufficiently increased a well-defined two-chain configuration is formed.

The time dependence of the MSD for a system with $n=1.0$ and $L / a=1$ is presented in Figs. 6(a) and 6(b), for $\Omega=10$ and $\Omega=100$, respectively. In both cases the STR is characterized by a normal diffusion behavior, i.e., $\left\langle\Delta r^{2}(t)\right\rangle \propto t^{1}$.

For $\Omega=10$, the diffusion in the ITR is not modified by increasing the substrate strength until $V_{0}=0.1$ [Fig. 6(a)], but after further increasing the substrate strength, the diffusion is enhanced, reaching a maximum at $V_{0}=0.16$. Such behavior is due to a structural transition, in which the system changes from a staggered to an aligned two-chain configuration. In the latter the particles are now pairwise aligned along the $y$ direction (and do not interlock with each other anymore). As a consequence, the particles in both chains are more free to move with respect to each other, resulting in a larger diffusion. Notice that in the case of staggered chains, the dynamic friction of the chains with respect to each other will be higher because of an interlocking effect. If one moves these chains far enough from each other, they will no longer be able to interlock and consequently they will slide much more easily over each other. A further increase of $V_{0}$ results in a larger correlation between the particles of distinct chains, resulting 

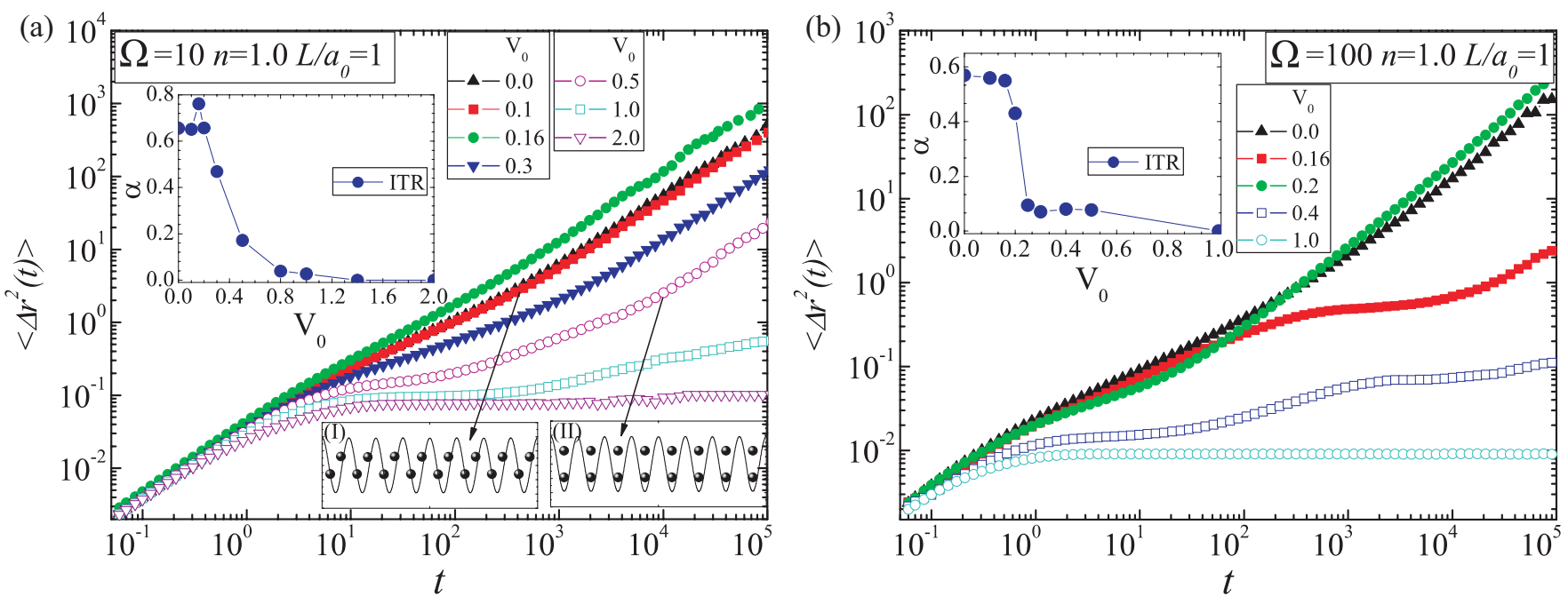

FIG. 6. (Color online) Log-log plot of the MSD curves taking into account both $x$ and $y$ directions, plotted as a function of time for $n=1.0$ and $L / a_{0}=1.0$ considering (a) $\Omega=10$ and (b) $\Omega=100$. The power-law coefficient $\alpha$ for the ITR is plotted as a function of $V_{0}$ in the upper insets, for $\Omega=10$ and $\Omega=100$. The lower insets of panel (a) show the ground state configuration for two values of $V_{0}$.

in a very slow diffusion. For $V_{0}>1.4$, diffusion is severely suppressed.

For long times (LTR), the normal diffusive regime is recovered for $V_{0} \leqslant 0.5$, while for $V_{0}>1.5$ the diffusion is completely suppressed in all directions. As an illustration we present the case $V_{0}=2.0$ [see Fig. 6(a)], where all diffusion is suppressed.

For $\Omega=100$ [Fig. 6(b)] we find initially $\left(V_{0}=0\right)$ that the MSD curve is characterized by a subdiffusive behavior, $\left\langle\Delta r^{2}(t)\right\rangle \propto t^{1 / 2}$, which identifies the SFD. For $V_{0}>0.16$, the slope of the MSD curves, and the corresponding $\alpha$ value, decreases with increasing $V_{0}$ [inset in Fig. 6(b)]. For $V_{0}>0.3$, particles in both chains become pinned at the minima of the substrate and the diffusion of the particles is completely suppressed.

In the LTR we observe that for $V_{0}=0$ normal diffusion is recovered, while for $0<V_{0}<0.16$ the diffusion is suppressed. However, for $V_{0} \geqslant 0.16$ [Fig. 6(b)], we found a remarkable enhancement of the diffusion. The physical reason is that the system undergoes a structural transition from the staggered configuration to an aligned configuration around $V_{0} \geqslant 0.16$, resulting in more freedom for particles to move along the channel.

To understand the effect of the substrate on the particle dynamics, we also plotted the MSD curves in the $x$ and $y$ directions separately [Figs. 7(a) and 7(b)]. For both $\Omega=10$ and $\Omega=100$ at short times (STR) normal diffusion occurs. At intermediate times (ITR), the diffusion process becomes subdiffusive while at sufficiently long times (LTR) diffusion is recovered for lower values of $V_{0}$. For larger $V_{0}$, diffusion is completely suppressed. From these curves one can see that diffusion in the $x$ direction occurs at time scales different than those of the one along the $y$ direction.

Note that for $\Omega=10$ and $V_{0} \approx 1.0$, the diffusion along the $x$ and $y$ directions [Fig. 7(a)] is suppressed in the ITR, but for the LTR diffusion recovers and particles start to diffuse in both directions again. This can be explained because the
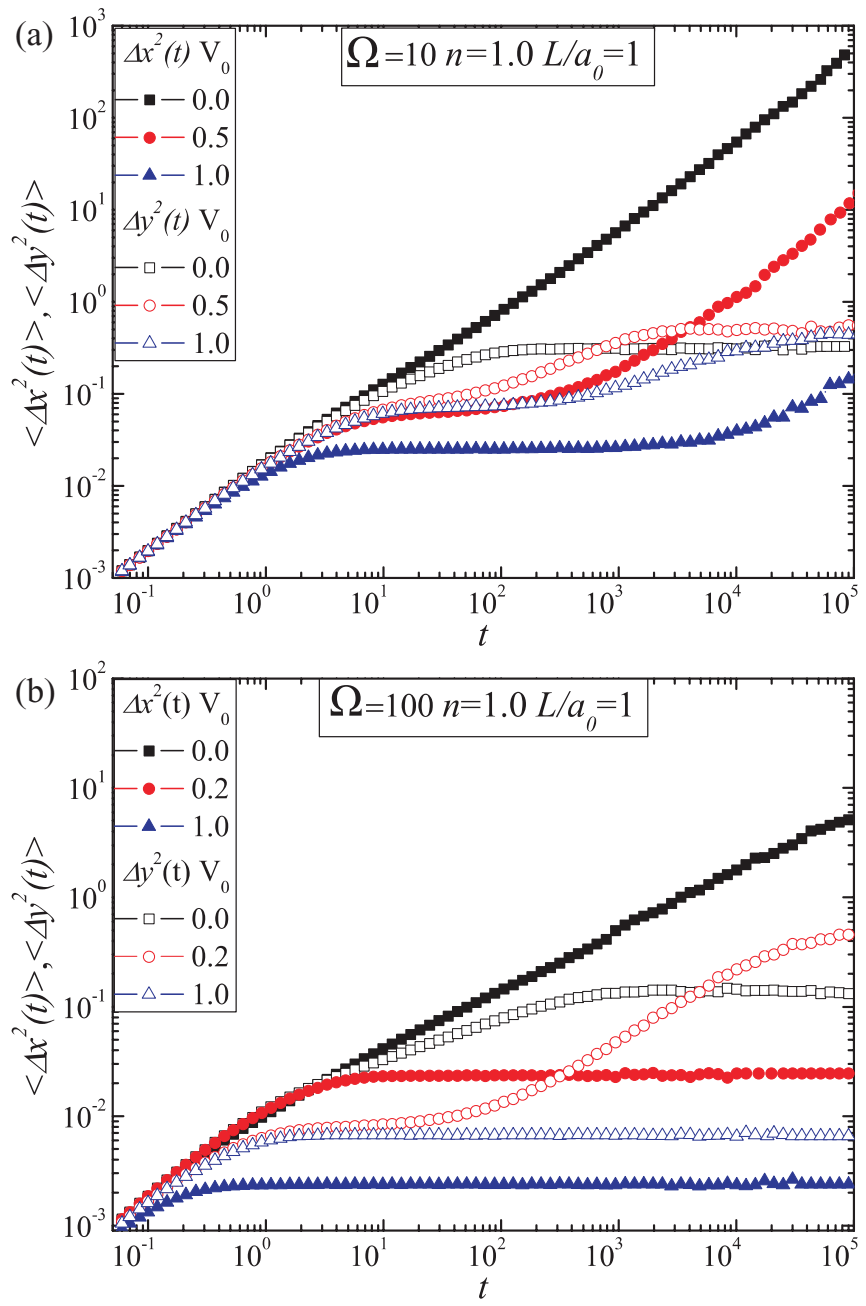

FIG. 7. (Color online) Log-log plot of the MSD curves in (a) the $x$ direction and (b) the $y$ direction for different values of the substrate strength, and for $\Omega=10$ and $\Omega=100$, respectively. 
particles oscillate around the substrate minima for a long time before reaching diffusive motion.

After a long period of time the particles inside each minimum of the periodic substrate eventually can change their positions depending on the strength of the substrate. As an example of such a behavior, we show the case for $\Omega=100$ and $V_{0} \approx 0.2$ [Fig. 7(b)]. Here the diffusion along the $x$ and $y$ directions is suppressed in the ITR, but for the LTR, the diffusion is recovered only in the $y$ direction. However for $V_{0}>0.3$, the particles become pinned even in the LTR.

From this subsection we can conclude that in a two-chain configuration, the diffusion in the $x$ direction occurs on a time scale different than that of the diffusion in the $y$ direction, resulting in a two-step diffusion process in the intermediate time diffusion regime. Further, an enhancement of the diffusion process is found for certain values of the substrate strength, which originates from a structural transition from a staggered to an aligned structure.

\section{The transition from the single-chain to the two-chain configuration}

To conclude, we study the case $n=0.5$ and $L / a_{0}=2.0$, where we found earlier a first-order structural transition from the single-chain to the two-chain regime with increasing $V_{0}$. For small $V_{0}$ values, the particles are located at the zeros of the substrate potential, resulting in a single-chain configuration. The separation between particles within the minima decreases with increasing $V_{0}$, while the system is found in the single-chain configuration. For the critical value $V_{0} \approx 0.8$, a two-chain configuration is reached through a first-order transition. In the two-chain regime the particles are aligned along the $y$ direction in each minimum of the substrate. In Figs. 3(c) and 3(d), we show the probability distributions of finding a particle at a certain position in the channel for $n=0.5$ and $L / a_{0}=2.0$ for $\Omega=100(T \neq 0)$. One can clearly see that the configuration depends on the substrate strength. If the substrate strength is increased, the particles undergo a structural transition from the single-chain to the two-chain configuration.

In Figs. 8(a) and 8(b), the MSD as a function of time for the system with $n=0.5$ and $L / a_{0}=2.0$ is presented for $\Omega=10$ and $\Omega=100$. In the ITR, a $\Omega$-dependent behavior is found for the mean-squared displacement. For $\Omega=10$ [Fig. 8(a)], an expected decrease of the diffusion is observed when $V_{0}$ is increased. The system goes from a single-file regime $\left(V_{0}=0\right)$ to an even more subdiffusive regime with increasing $V_{0}$. In the inset of Fig. 8(a), one can see that $\alpha$ reduces with increasing substrate strength from $\alpha=0.5$ to $\alpha=0.3$, where it reaches a subdiffusive behavior.

For $\Omega=100$ a reentrant behavior is found for the diffusion in the ITR as a function of $V_{0}$. First, as expected, the diffusion in the ITR is reduced by increasing the substrate strength until $V_{0}=0.4$ [Fig. 8(b)]. With a further increase of the substrate strength, the diffusion is enhanced, reaching a new maximum for $V_{0} \approx 0.7$. The reason is that the system will go through a structural transition from the single-chain to the two-chain configuration. Since particles are now organized in two chains, the average distance between two neighboring particles along each chain is increased, resulting in a larger diffusion. After the two-chain configuration is formed, a further increase of $V_{0}$ will lead to a reduction of the diffusion because particles become more trapped at the potential minima. Finally, for $V_{0} \geqslant 1.3$, the diffusion is completely suppressed again. However this is not observed for $\Omega=10$, since for small $\Omega$ the particles have much more thermal energy, which allows them to escape from the substrate minima and prevents them from becoming pinned when the substrate amplitude $V_{0}<0.8$.

From this subsection we can conclude that the diffusion can be enhanced by a slight increase of the substrate potential because of a structural transition from the staggered to the aligned state. Further, a reentrant behavior of the MSD as a function of the substrate strength is found in the strongly coupled case $(\Omega=100)$. The reentrant behavior is induced by a structural phase transition from the single-chain to the two-chain configuration.
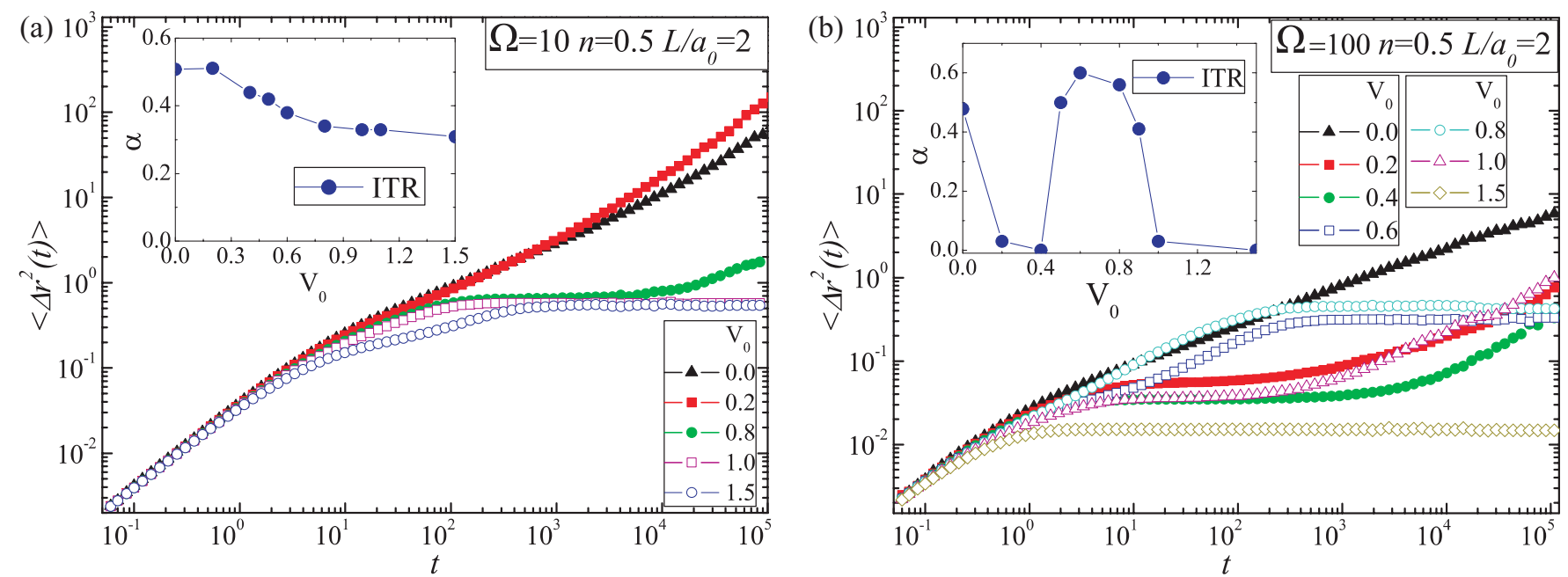

FIG. 8. (Color online) Log-log plot of the MSD curves taking into account both $x$ and $y$ directions, plotted as a function of time for $n=0.5$ and $L / a_{0}=2.0$ considering (a) $\Omega=10$ and (b) $\Omega=100$. The power-law coefficient $\alpha$ for the ITR is plotted as a function of $V_{0}$ in the insets, for $\Omega=10$ and $\Omega=100$, respectively. 


\section{CONCLUSIONS}

We used Brownian dynamic simulations to study the diffusive motion of charged particles in a Q1D colloidal system subjected to a sinusoidal substrate potential. In particular, we calculated the MSD for different substrate strengths. The simulations were performed for three different ground state configurations: (i) the single-chain configuration, (ii) the two-chain configuration, and (iii) a substrate-dependent configuration, where the system undergoes a structural transition from the single-chain to the two-chain configuration as a function of the substrate potential.

In general, we can conclude that the substrate potential will reduce the mobility of the particles, which for a critical value of the substrate strength leads to the pinning of particles at the minima of the substrate potential. The critical value of $V_{0}$ depends sensitively on the value of the parameter $\Omega$. Larger $\Omega$ values leads to smaller critical values of $V_{0}$.

In the case where particles are organized in a two-chain configuration, the diffusion in the $x$ and $y$ directions have different time scales, resulting in a two-step diffusion process in the intermediate diffusion regime. Further, an enhancement of the diffusion process can be found for certain values of the substrate strength, which is originated from a structural transition from the staggered to the aligned configuration.

If the configuration of the particles depends on the strength of the substrate potential, structural transitions will have a larger impact on the diffusive behavior of the particles. Here we showed that the diffusion of the particles can be enhanced by an increase of the substrate strength because of a structural transition from a staggered to an aligned configuration. Furthermore, a reentrant behavior of the MSD as a function of the substrate potential was found for strongly coupled systems. This reentrant behavior is induced by a structural transition from the single-chain to the two-chain configuration.

\section{ACKNOWLEDGMENTS}

This work was supported by the Brazilian agencies CNPq and FUNCAP (PRONEX-Grant), the Flemish Science Foundation (FWO-Vl), and the bilateral projects between Flanders and Brazil and the Flemish Science Foundation (FWO-Vl) and CNPq.
[1] A. Einstein, Ann. Phys. (Berlin) 17, 549 (1905).

[2] T. E. Harris, J. Appl. Probab. 2, 323 (1965).

[3] J. A. Hernandez and J. Fischbarg, J. Gen. Physiol. 99, 645 (1992).

[4] J. H. Morais-Cabral, Y. Zhou, and R. MacKinnon, Nature (London) 414, 37 (2001).

[5] D. A. Doyle, J. M. Cabral, A. K. Pfuetzner, J. M. Gulbis, S. L. Cohen, B. T. Chait, and R. MacKinnon, Science 280, 69 (1998).

[6] D. S. Sholl and K. A. Fichthorn, Phys. Rev. Lett. 79, 3569 (1997).

[7] H. Konig, R. Hund, K. Zahn, and G. Maret, Eur. Phys. J. E 18, 287 (2005).

[8] Q.-H. Wei, C. Bechinger, and P. Leiderer, Science 287, 625 (2000).

[9] K. Nelissen, V. R. Misko, and F. M. Peeters, Europhys. Lett. 80, 56004 (2007).

[10] N. Laachi, C. Declet, C. Matson, and K. D. Dorfman, Phys. Rev. Lett. 98, 098106 (2007).

[11] M. Kollmann, Phys. Rev. Lett. 90, 180602 (2003).

[12] V. Kukla, J. Kornatowski, D. Demuth, I. Girnus, H. Pfeifer, L. V. C. Rees, S. Schunk, K. K. Unger, and J. Karger, Science 272, 702 (1996).

[13] W. M. Meier and D. H. Olsen, eds., Atlas of Zeolite Structure Types (Butterworths-Heinemann, London, 1992).
[14] K. Hahn, J. Karger, and V. Kukla, Phys. Rev. Lett. 76, 2762 (1996).

[15] C. Lutz, M. Kollmann, and C. Bechinger, Phys. Rev. Lett. 93, 026001 (2004).

[16] C. Coste, J.-B. Delfau, C. Even, and M. Saint Jean, Phys. Rev. E 81, 051201 (2010).

[17] J.-B. Delfau, C. Coste, and M. Saint Jean, Phys. Rev. E 84, 011101 (2011).

[18] A. Taloni and M. A. Lomholt, Phys. Rev. E 78, 051116 (2008).

[19] M. Köppl, P. Henseler, A. Erbe, P. Nielaba, and P. Leiderer, Phys. Rev. Lett. 97, 208302 (2006).

[20] A. Homann, A. Melzer, S. Peters, and A. Piel, Phys. Rev. E 56, 7138 (1997).

[21] Bin Liu and J. Goree, Phys. Rev. E 71, 046410 (2005).

[22] J. C. N. Carvalho, W. P. Ferreira, G. A. Farias, and F. M. Peeters, Phys. Rev. B 83, 094109 (2011).

[23] D. L. Ermak and J. A. McCammon, J. Chem. Phys. 69, 1352 (1978).

[24] A. Taloni and F. Marchesoni, Phys. Rev. Lett. 96, 020601 (2006).

[25] S. Herrera-Velarde and R. Castaneda-Priego, Phys. Rev. E 79, 041407 (2009). 